\title{
Localization and Regulation of Polymeric Ig Receptor in Healthy and Diseased Human Kidney
}

Krzysztof M. Krawczyk, ${ }^{*}$ Helén Nilsson, ${ }^{*}$ Jenny Nyström, ${ }^{\dagger}$ David Lindgren, ${ }^{\ddagger}$ Karin Leandersson, ${ }^{\S}$ Karl Swärd, ${ }^{\natural}$ and Martin E. Johansson* ${ }^{*}$

\begin{abstract}
From the Centers for Molecular Pathology* and Cancer Immunology, ${ }^{\S}$ Department of Translational Medicine, Lund University, Malmö; the Institutes of Neuroscience and Physiology ${ }^{\dagger}$ and Biomedicine, ${ }^{\|}$University of Gothenburg, Gothenburg; and the Center for Translational Cancer Research, ${ }^{\ddagger}$ the Department of Laboratory Medicine, and the Department of Experimental Medical Science, "Lund University, Lund, Sweden
\end{abstract}

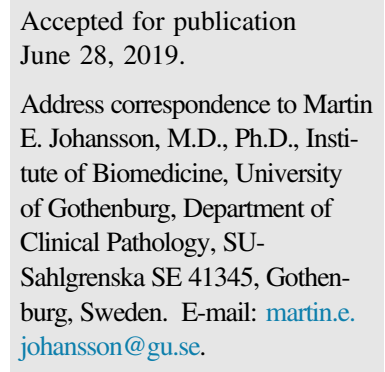

\begin{abstract}
The polymeric Ig receptor (PIgR) constitutes an important part of the immune system by mediating transcytosis of dimeric IgA into mucosal fluids. Although well studied in organs such as the intestine, the regulation and localization of PIgR in human kidney are incompletely characterized. Herein, using immunohistochemistry, we show that in healthy human kidneys, PIgR is expressed by the progenitorlike tubular scattered cells of the proximal tubules and by parietal epithelial cells of glomeruli. We further show that proximal tubular expression of PIgR becomes widespread during kidney disease, correlating to elevated levels of urinary secretory IgA. Urinary secretory IgA levels also correlated to the degree of tubular fibrosis, plasma creatinine, and urea levels. In addition, primary tubular cells were cultured to study the function and regulation of PIgR in vitro. Cellular PIgR expression was induced by conditioned medium from activated human leukocytes, as well as by inflammatory cytokines, whereas transforming growth factor- $\beta 1$ caused decreased expression. Furthermore, interferon- $\gamma$ increased the transcytosis of dimeric IgA in cultured tubular cells. Finally, a correlation study of mRNA data from the Genotype-Tissue Expression portal indicated that PIGR mRNA expression in kidney correlates to the expression of TNFSF13, a cytokine involved in plasma cell class switching to IgA. These results indicate that PIgR induction is an integral part of the injury phenotype of renal tubular cells. (Am J Pathol 2019, 189: 1933-1944; https://doi.org/10.1016/j.ajpath.2019.06.015)
\end{abstract}

The mucosal linings of the gastrointestinal and respiratory tracts are continuously bathed with secreted IgA. Secreted $\operatorname{IgA}$ is also an important component of breast milk and is found at low levels in urine. This mucosal immunity provides a barrier against external pathogens. ${ }^{1} \operatorname{IgA}$ is carried across the mucosal epithelium by the polymeric Ig receptor (PIgR), localized in the basolateral plasma membranes of the epithelial cells. Plasma cells in the underlying lamina propria produce dimeric $\operatorname{IgA}(\mathrm{dIgA})$ by joining the Fc regions of two $\operatorname{IgA}$ molecules with a protein called joining chain (JC). The JC is recognized by PIgR that mediates transcellular transport of IgA to the apical compartment, where $\operatorname{dIgA}$ is cleaved off from PIgR, leaving a piece of PIgR called secretory component (SC) covalently attached to the secreted $\operatorname{dIgA}$ (Figure 1). The resulting molecule is called secretory $\operatorname{IgA}(\operatorname{sIg} \mathrm{A})$. Human urine also contains
$\mathrm{sIgA}^{2}$; and as early as in 1969 , Tourville et $\mathrm{al}^{3}$ reported PIgR/SC localization to unspecified kidney tubules of some, but not all, human kidneys. Another early study also reported that some tubules were positive for PIgR/SC, but neither of these studies provided conclusive histology or regulatory data. ${ }^{4}$

The cellular basis for kidney regeneration after injury is still under debate. The classic view of randomly surviving

\footnotetext{
Supported by a Marianne \& Marcus Wallenberg Foundation grant, a National Association against Kidney Diseases grant, the Professor LarsErik Gelins Commemorative fund, National Health Service governmental funding of clinical research, Skånes Universitetssjukhus foundations and donations, the Malmö General Hospital Research Fund for cancer research, the Strategic Cancer Research Program Biocare, and the Swedish Cancer Society (all to M.E.J.).

Disclosures: None declared.
} 

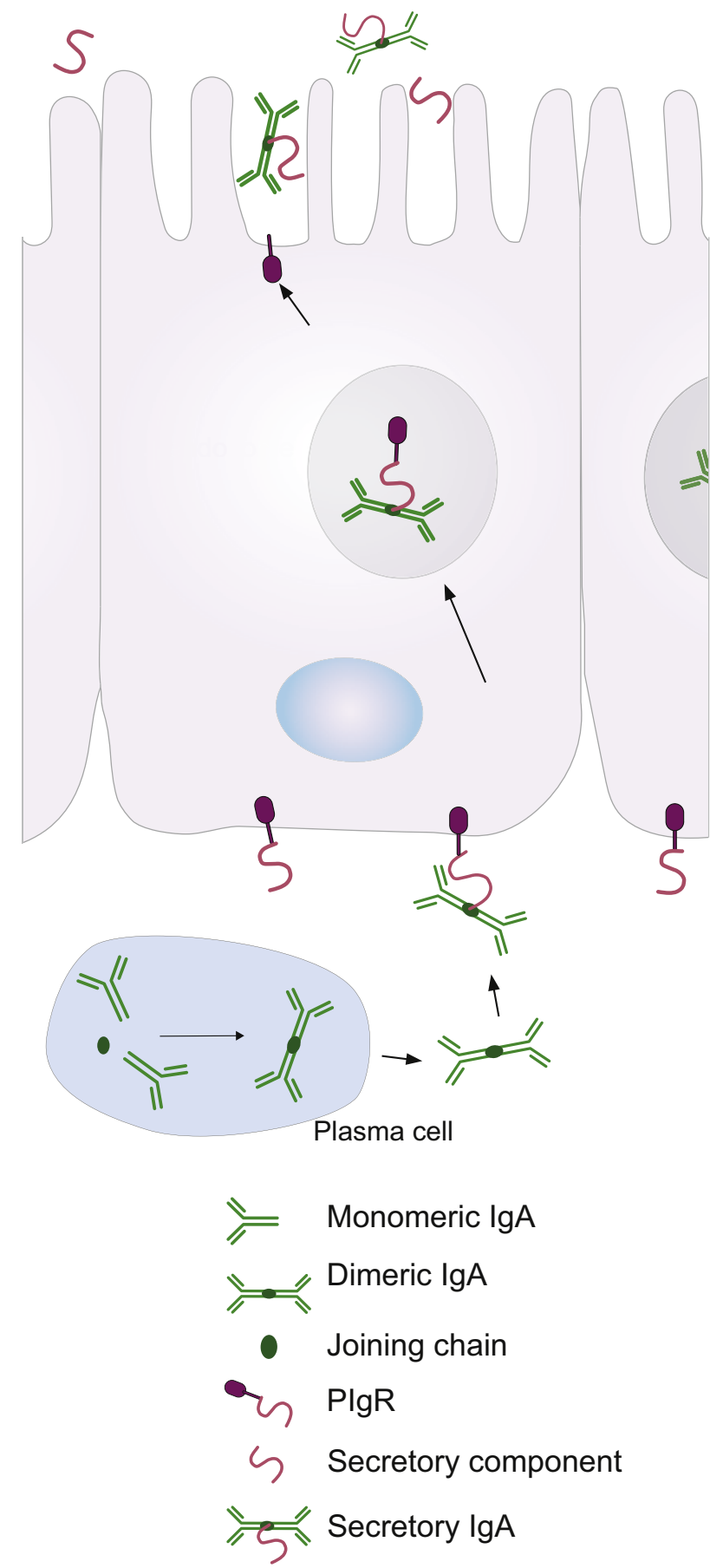

Figure 1 Schematic illustration of dimeric IgA transcytosis by polymeric Ig receptor (PIgR). Mucosa-associated plasma cells produce dimeric IgA by joining two monomeric IgA molecules with a joining chain. Dimeric IgA is recognized by PIgR in the basolateral plasma membrane of mucosal epithelia and undergoes endocytosis and transport via the endosomal compartment to the apical surface. There, dimeric IgA is cleaved off from PIgR, leaving a part of the PIgR molecule, called secretory component, covalently attached to the dimeric IgA, generating the secretory IgA.

cells undergoing epithelial-to-mesenchymal transition has been challenged by the concept of a renal progenitor cell population mediating repair. ${ }^{5-9}$ Regardless, a population of tubular cells with bearing on injury and regeneration has been identified scattered throughout the proximal tubules [tubular scattered cells (TSCs)], and these increase in number after injury. ${ }^{6,8}$ Herein, we show that PIgR is expressed by the TSCs in healthy proximal tubules. PIgR expression becomes widespread in injured kidneys, and urinary sIgA levels increase in patients displaying a broad set of kidney diseases. Furthermore, we show that PIgR expression is induced in cultured primary human tubular cells by inflammatory cytokines or conditioned medium from human leukocytes. We also show in vitro that cultured tubular cells perform transcytosis of IgA and that this process is stimulated by interferon- $\gamma$ (IFN- $\gamma$ ). These results indicate that PIgR expression is part of the tubular response to injury, providing an explanation for the increased $\operatorname{sIg} \mathrm{A}$ levels found in urine from injured kidneys.

\section{Materials and Methods}

\section{Patient Material}

Histologic material, plasma, and urine samples were obtained after informed consent from healthy individuals and patients. Ethical permit (number 413-09) was approved by the ethical committee at University of Gothenburg (Gothenburg, Sweden). Clinical data are summarized in Table 1. Tissue was also obtained from kidneys removed by nephrectomy after patient informed consent and with ethical permission from the Regional Ethics Committee at Lund University (Lund, Sweden; LU680-08 and LU289-07). Leukocytes from healthy blood donors were isolated from leukocyte concentrates, and permission for this was obtained from the Regional Ethics Committee at Lund University (LU949-17). The participating individuals provided a written informed consent before the study.

\section{Immunohistochemistry and Histochemistry}

Immunohistochemical analysis was performed on sections (4 $\mu \mathrm{m}$ thick) of formalin-fixed, paraffin-embedded kidney tissue. Deparaffinization and epitope retrieval were performed in a PT Link module (Dako, Stockholm, Sweden), and staining was done using Autostainer Plus equipment (Dako), according to the manufacturer's protocols. The following antibodies were used: anti-secretory component of PIgR (sc-20656; dilution 1:200; Santa Cruz Biotechnology, Dallas, TX), anti-vimentin (M0725; dilution 1:1000; Dako), anti-IgJ (sc-133177; dilution 1:50; Santa Cruz Biotechnology), and anti-plasma cell marker (M7077; dilution 1:50; Dako).

Grading the extent of PIgR expression in the proximal tubules and degree of tissue fibrosis was performed by a subspecialized kidney pathologist (M.E.J.). The evaluated tissue sections were from the same patients who were included in the present study (Table 1). The extent of fibrosis was evaluated by assessment of the percentage of trichrome blue areas in diseased kidney compared with 
Table 1 Summary of Patient Data

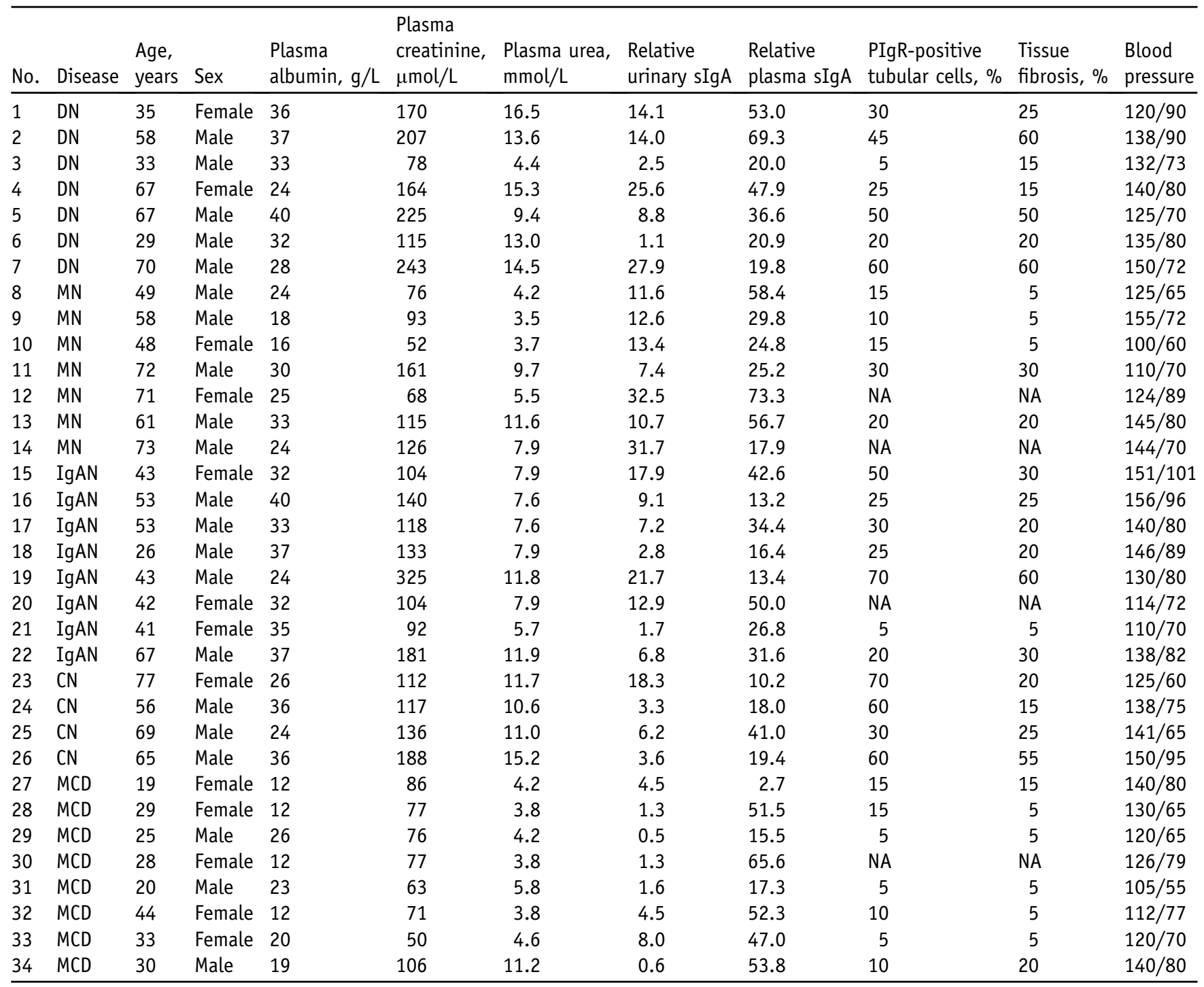

CN, crescentic glomerulonephritis; DN, diabetic nephropathy; IgAN, IgA nephropathy; MCD, minimal change disease; MN, membranous nephropathy; NA, tissue not available; PIgR, polymeric Ig receptor; $\operatorname{sIgA}$, secretory IgA.

control material. Trichrome staining was performed using the Dako ArtisanLink system and Masson's Trichrome staining kit AR173 (both from Dako), according to the manufacturer's protocols.

\section{Immunofluorescence and Colocalization Studies}

For colocalization studies, immunofluorescence was performed on formalin-fixed, paraffin-embedded material from four healthy individuals and three patients with diabetic nephropathy. After deparaffinization, antigen retrieval was performed in DIVA decloaker (Histolab, Gothenburg, Sweden). The antibodies used were mouse anti-vimentin (ab8978; dilution 1:200; Abcam, Cambridge, UK), rabbit anti-secretory component of PIgR (sc-20656; dilution 1:200; Santa Cruz Biotechnology). Secondary anti-mouse and anti-rabbit antibodies conjugated to Alexa Fluor 488 or Alexa Fluor 594, from Thermo Fisher Scientific (Waltham, MA), were used. Sections were mounted with mounting medium containing DAPI (Vector Laboratories, Burlingame, CA). For assessment of $\operatorname{IgA}$ and $\operatorname{IgG}$ distribution in atrophic tubules, unfixed cryosections were produced, according to standard protocol, and stained with fluorescein isothiocyanate-labeled antisera against IgA or IgG (Dako).

\section{Sandwich Enzyme-Linked Immunosorbent Assay for Measurement of Secretory IgA}

Secretory $\operatorname{IgA}$ in plasma and urine was analyzed by sandwich enzyme-linked immunosorbent assay in 96-well microplates (Greiner bio-one, Courtaboeuf Cedex, France). Wells were coated with mouse anti-SC antibody (I-6635; 
Sigma-Aldrich, St. Louis, MO) and blocked with phosphate-buffered saline with Tween-20 containing $1 \%$ (w/v) bovine serum albumin. Urine and blood samples were added to the coated wells in triplicates. Secretory IgA was detected using a rabbit anti-human IgA antibody (A0262; Dako), followed by anti-rabbit horseradish peroxidase-linked antibody (NA934V; GE Healthcare, Little Chalfont, UK). O-phenylenediamine was added as a substrate, and plates were incubated for 30 minutes in darkness at room temperature. The reaction was stopped by addition of $3 \mathrm{~mol} / \mathrm{L}$ sulfuric acid, and absorbance was measured at $492 \mathrm{~nm}$ on a FLUOstar Omega plate reader (BMG Labtech, Värmdö, Sweden). For standard curve preparation, purified human secretory IgA (55905; MP Biomedicals, Santa Ana, CA) was used. Urine sIgA levels were normalized to urine creatinine levels.

\section{Cell Culture and Treatments}

Tubular cell cultures were prepared according to previously published protocols. ${ }^{10}$ In short, cortical kidney tissue was minced and dissociated in a collagenase type I and DNase I type II solution. Single cells were seeded and cultured in Dulbecco's modified Eagle's medium low glucose with $10 \%$ fetal bovine serum and $1 \%$ penicillin-streptomycin (Thermo Fisher Scientific) at $37^{\circ} \mathrm{C}$ and $5 \% \mathrm{CO}_{2}$. Cultured cells were treated with recombinant IFN- $\gamma$ (285-IF; R\&D Systems, Minneapolis, MN) at $12.5 \mathrm{ng} / \mathrm{mL}$, transforming growth factor- $\beta 1$ (240-B; R\&D Systems) at $10 \mathrm{ng} / \mathrm{mL}$, IL$1 \beta$ (201-LB; R\&D Systems) at $25 \mathrm{ng} / \mathrm{mL}$, or tumor necrosis factor- $\alpha$ (TNF- $\alpha$; 8902SF; Cell Signaling, Danvers, MA) at $10 \mathrm{ng} / \mathrm{mL}$.

Peripheral blood mononuclear cells were isolated from leukocyte depletion filters from healthy donors, according to a previous publication. ${ }^{11}$ Conditioned medium from peripheral blood mononuclear cells was collected after 24 hours of culture in Dulbecco's modified Eagle's medium low glucose with $10 \%$ heat-inactivated human serum (Sigma-Aldrich) and 1\% penicillin-streptomycin solution. To obtain conditioned medium from activated immune cells, $100 \mathrm{ng} / \mathrm{mL}$ of lipopolysaccharides from Escherichia coli (Sigma-Aldrich) was added to the culture medium.

\section{Real-Time Quantitative PCR}

The mRNA levels of PIGR were measured by real-time quantitative PCR. RNA was isolated from cultured cells using the RNeasy mini kit (Qiagen, Hilden, Germany). cDNA was generated using the RevertAid RT kit (Thermo Fisher Scientific). Real-time quantitative PCRs were prepared in triplicates and performed on a Stratagene Mx3005P (Agilent Technologies, Santa Clara, CA). Results were normalized using two housekeeping genes: HMBS, 5'-GGCAATGCGGCTGCAA- $3^{\prime}$ (forward primer) and 5'-GGGTACCCACGCGAATCAC- $3^{\prime}$ (reverse primer); and RPLI3A, 5'-CCTGGAGGAGAAGAGGAAAGAGA- $3^{\prime}$ (forward primer) and $5^{\prime}$ -
TTGAGGACCTCTGTGTATTTGTCAA-3' (reverse primer). Primer sequences for PIGR were as follows: $5^{\prime}$ GCCCGAGCTGGTTTATGAAG- $3^{\prime}$ (forward) and $5^{\prime}$ AGCCGTGACATTCCCTGGTA-3' (reverse).

\section{Western Blot Analysis}

Cultured cells were harvested and lysed in radioimmunoprecipitation assay buffer supplemented with complete protease inhibitor cocktail (Sigma-Aldrich). Western blot analysis was performed according to the manufacturer's protocol using precast gels, polyvinylidene difluoride membranes, and Trans-blot Turbo transfer from Bio-Rad Laboratories (Hercules, CA). Antibodies used were anti- $\beta$-actin (dilution 1:5000; Sigma-Aldrich) and anti-PIgR/SC (dilution 1:1000; Santa Cruz Biotechnology). Secondary horseradish peroxidase-conjugated anti-mouse and anti-rabbit antibodies (GE Healthcare) were used, and protein bands were visualized with Western blot ECL (AH Diagnostics, Solna, Sweden) and a ChemiDoc camera (Bio-Rad Laboratories).

\section{Secretory IgA Transport Study}

For IgA transport study, tubular cells were seeded on $0.4-\mu \mathrm{m}$ pore membrane inserts placed in 6-well plates (Corning, Corning, NY) and cultured until confluency. Purified human IgA (P80-102; Bethyl Laboratories, Montgomery, TX) was added to the lower compartment. After 18 hours, medium from both compartments was collected and analyzed for secretory IgA content using sandwich enzymelinked immunosorbent assay, as described above. For PIgR induction, cells were treated with $12.5 \mathrm{ng} / \mathrm{mL} \mathrm{IFN-} \gamma$ for 48 hours before the transport study.

\section{RNA Expression Correlation Study}

RNA-sequencing data from the Genotype-Tissue Expression (GTEx) project (http://www.gtexportal.org) ${ }^{12}$ were downloaded in August 2016. After normalization according to the trimmed mean of $\mathrm{M}$-values method, ${ }^{13}$ correlation analyses were made using the Pearson method. Correlation between PIGR and all other transcripts $(56,202)$ was analyzed in the eight tissue types with highest PIGR levels ( $n=57$, salivary gland; $n=196$, transverse colon; $n=88$, terminal ileum; $n=32$, kidney; $n=320$, lung; $n=193$, stomach; $n=119$, liver; $n=214$, mammary tissue). The sum of correlation coefficients across tissues for all individual RNAs [Rsum (versus PIGR)] was calculated. This sum was sorted in descending order, and the extremes were plotted. Correlations that seemed biologically meaningful [tumor necrosis factor ligand superfamily member 13 (TNFSF13), TNFRSF11A, and STATI] were next tested using the Spearman method (GraphPad Prism software version 8; GraphPad Software, San Diego, CA) in kidney ( $n$ $=32 ;$ ie, the smallest data set). 


\section{Statistical Analysis}

GraphPad Prism software was used for statistical analyses. Pairwise comparisons were made using a paired, two-tailed $t$-test. For multiple comparisons, one-way analysis of variance was used, followed by Dunnett's correction for multiple comparisons. Correlations were calculated using Pearson's method.

\section{Results}

The Expression Pattern of PIgR in Healthy Human Kidney Is Similar to that of the TSC Marker Vimentin and Increases after Injury

To determine the presence and localization of PIgR in human kidney, healthy kidney tissue was analyzed by immunohistochemistry. Healthy glomeruli and proximal tubules are visualized by hematoxylin and eosin staining in Figure 2A. Scattered PIgR positivity was detected in the proximal tubules and in the parietal epithelial cells of Bowman capsule (Figure 2B). This staining pattern is similar to what has been previously observed for TSC markers, such as vimentin and CD133. ${ }^{6}$ Vimentin is expressed by kidney stromal cells, endothelial cells, and parietal epithelial cells, but apart from the presence in the TSCs, it is not normally expressed in proximal tubules
(Figure 2C). Figure 2D demonstrates the morphology of kidney tissue affected by diabetic nephropathy, with flattened proximal tubular cells and visible interstitial fibrosis. The expression of TSC markers becomes extensive following injury in proximal tubules. ${ }^{6,8}$ Similarly, widespread PIgR expression was found in the proximal tubules of the diseased kidney (Figure 2E). Thus, PIgR shows an overlapping expression pattern in the healthy and diseased kidney with the TSC marker vimentin (Figure 2, C and F).

\section{PIgR Colocalizes with Vimentin in Healthy and Diseased Kidney}

Having established a similar expression pattern of vimentin and PIgR in human healthy and diseased kidney, a potential coexpression of these two markers was studied. Immunofluorescence costaining of vimentin and PIgR shows overlapping staining patterns both in TSCs and in parietal epithelial cells of healthy kidneys (Figure 3, A-D). A slightly higher number of tubular cells were positive for vimentin, but approximately $80 \%$ of the tubular cells that were positive for vimentin also expressed PIgR. Expression of both markers was more widespread in diseased kidney (Figure 3, E-H), and here the degree of colocalization was even higher.
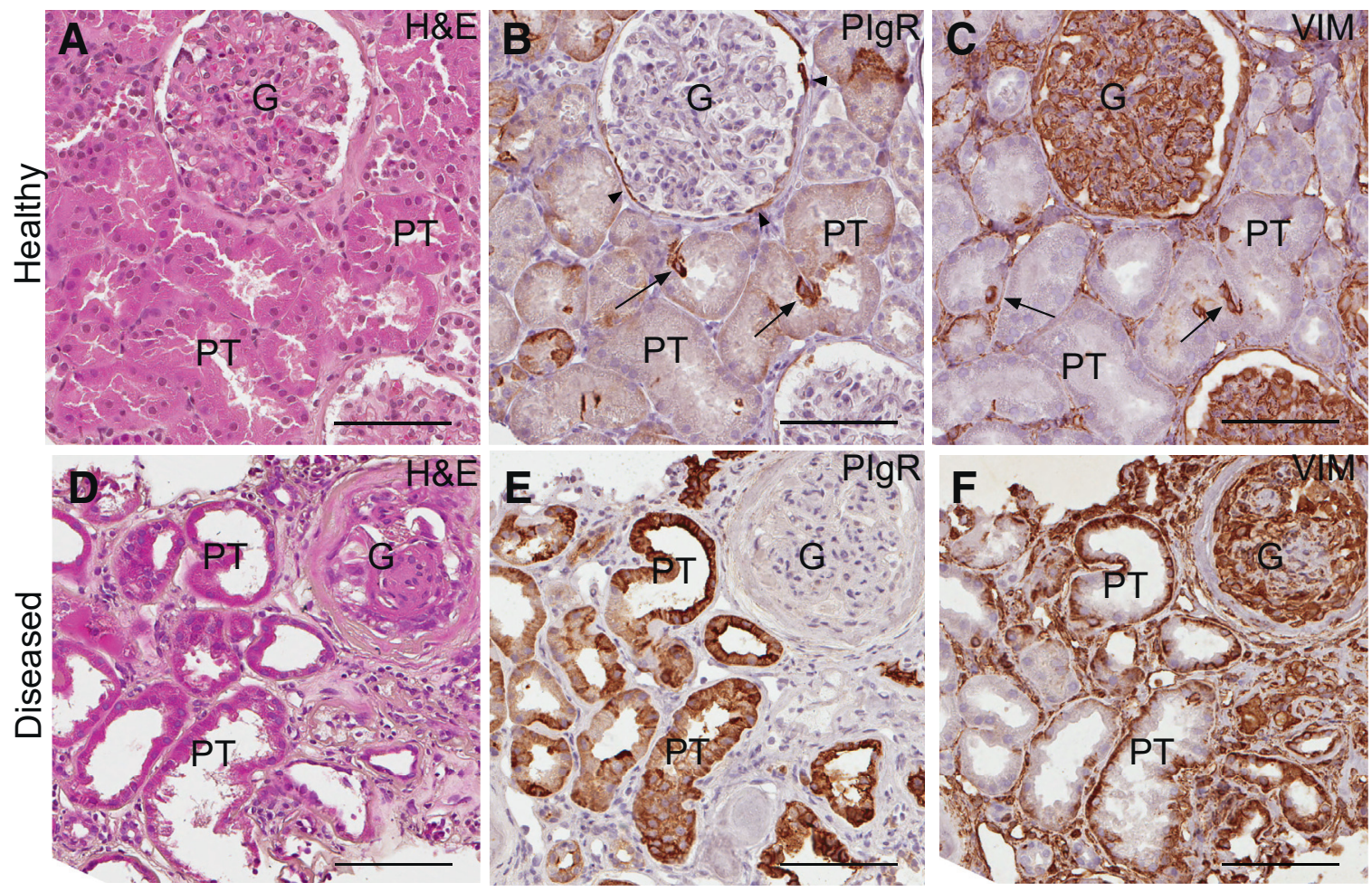

Figure 2 Polymeric Ig receptor (PIgR) and vimentin (VIM) expression in healthy and diseased human kidney. A: Hematoxylin and eosin (H\&E) staining of healthy kidney tissue showing normal kidney morphology. B and C: Immunohistochemical PIgR (B) and vimentin (C) staining of serial sections from the same normal kidney tissue, showing PIgR expression in the parietal epithelial cells of Bowman capsule (arrowheads) and in scattered cells present in the proximal tubules (PTs; arrows). VIM staining demonstrates scattered positive cells in the proximal tubules (C). D-F: H\&E staining (D) of chronically injured kidney tissue from a patient with diabetic nephropathy (consecutive sections; D-F), showing interstitial fibrosis with flattened proximal tubular cells highly positive for PIgR (E) and vimentin (F). $n=4$. Scale bars $=100 \mu \mathrm{m}(\mathbf{A}-\mathbf{F}) . \mathrm{G}$, glomerulus. 

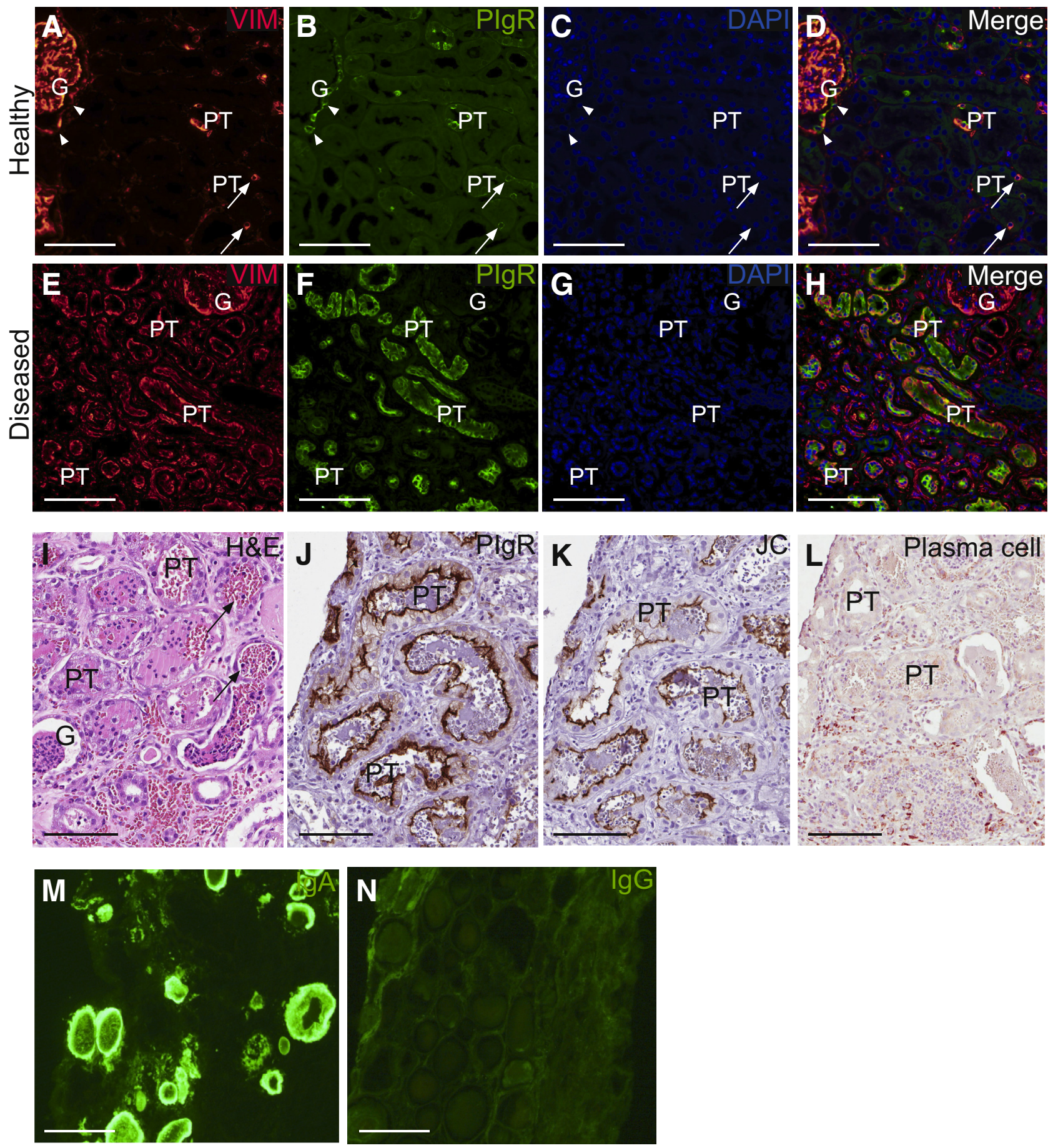

Figure 3 Polymeric Ig receptor (PIgR) and vimentin (VIM) are colocalized in healthy and diseased human kidney. A-H: Immunofluorescence costaining of vimentin (red) and PIgR (green) counterstained with DAPI (blue) shows double positivity in parietal epithelial cells (arrowheads) and in scattered cells in proximal tubules (PTs; arrows) of healthy (A-D) and diseased (E-H) kidney. Costaining was performed in tissue from four healthy individuals and three with diabetic nephropathy. I-L: Consecutive sections from a pyelonephritis case. Hematoxylin and eosin (H\&E) staining showing flattened proximal tubules (arrows) filled with erythrocytes and neutrophils is shown (I). J-L: The injured proximal tubules demonstrate apical PIgR positivity (J) and distinct joining chain (JC) positivity $(\mathbf{K})$; however, the plasma cells $(\mathbf{L})$ are negative for $\mathrm{JC}(\mathbf{K})$. $\mathbf{M}$ and $\mathbf{N}$ : Immunofluorescence staining of a case of nephrosclerosis shows IgA-positive $(\mathbf{M})$, but IgG-negative $(\mathbf{N})$, proteinaceous material in the lumen of the chronically injured proximal tubules, demonstrating so-called thyroidization phenomenon as a histologic sign of tubular atrophy. Scale bars $=100 \mu \mathrm{m}(\mathbf{A}-\mathbf{N}) . \mathrm{G}$, glomerulus.

PIgR expression was further analyzed in kidney tissue from a pyelonephritis patient displaying heavy injury with blood present in the lumen of proximal tubules (Figure 3I). The injured proximal tubules show widespread apical PIgR staining (Figure $3 \mathrm{~J}$ ). We could also demonstrate the presence of JC in the lumen of these proximal tubules (Figure $3 \mathrm{~K}$ ), indicating the presence of dIgA. Of note, the infiltrating plasma cells found in the interstitium of these 


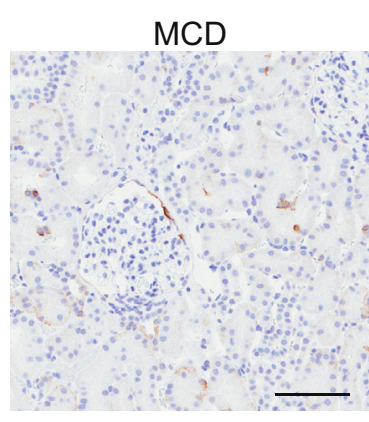

MN

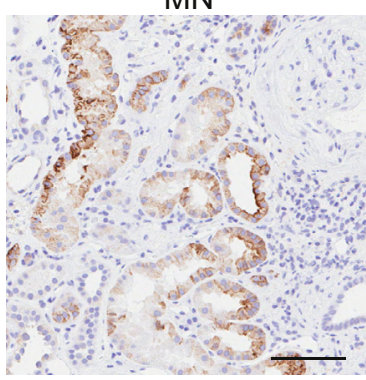

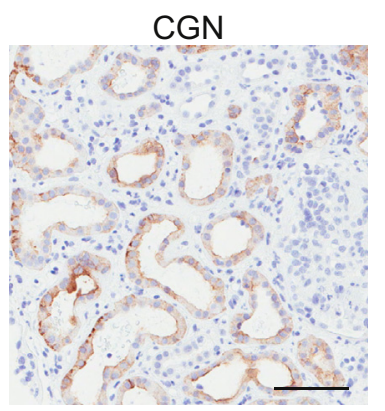

DN

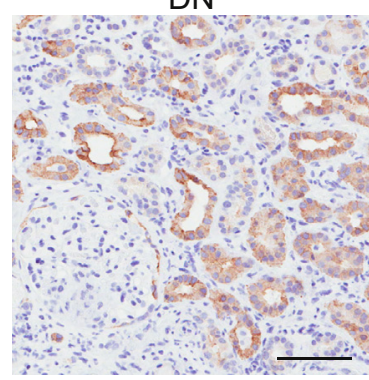

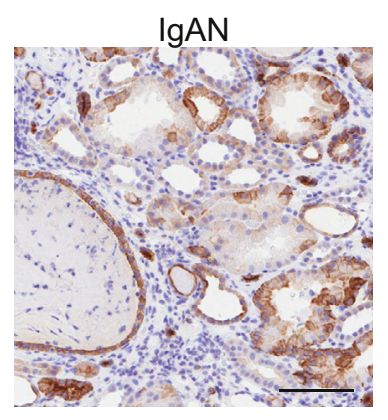

Figure 4 Polymeric Ig receptor (PIgR) expression correlates to disease severity. Immunohistochemical staining of histologic material from kidney biopsies obtained from patients experiencing the indicated kidney diseases. With the exception of minimal change disease (MCD), increased PIgR expression can be observed in kidney tissue affected by the diseases. Representative sections from each disease are shown. The number of patients in each group were as follows. $n=7 \mathrm{MCD}$, IgA nephropathy (IgAN), and diabetic nephropathy (DN); $n=4$ crescentic glomerulonephritis (CGN); $n=5$ membranous nephropathy (MN). Scale bars $=100 \mu \mathrm{m}$ kidneys (Figure 3L) were not JC positive (Figure 3K), which might indicate that the dIgA was not produced locally. Finally, immunofluorescence staining for $\operatorname{IgA}$ showed positivity in proteinaceous deposits found in the lumen of chronically injured proximal tubules (Figure 3M). In contrast, these deposits were negative for IgG (Figure $3 \mathrm{~N}$ ) that is present in large amounts in blood but is not transported by PIgR. This pattern is seen in chronically injured kidney tissue, where fibrosis is increased, regardless of underlying disease. These results indicate that active PIgR-mediated IgA transport occurs in injured proximal tubules and further support a functional role of increased PIgR expression during chronic kidney injury.

PIgR Expression and Urine sIgA Levels Correlate to the Degree of Kidney Injury

To get a more complete understanding of the extent of PIgR expression in different forms of kidney disease, histologic samples from a cohort of 34 patients experiencing various kidney conditions were analyzed (patient data presented in Table 1). PIgR expression and tissue fibrosis, as determined by Masson's trichrome staining, were quantified for all patients from whom tissue was available. Examples of immunohistochemical staining of PIgR from the different conditions are shown in Figure 4. Interestingly, proximal tubule PIgR expression seems to increase with the severity of the disease. A significant positive correlation was found between the percentage of PIgR-positive tubular cells and the extent of tissue fibrosis across all conditions. Tubular PIgR expression was also significantly correlated to plasma creatinine and urea levels, which are classic analytes used to assess kidney injury (Table 2).

To determine whether the observed induction of PIgR expression in injured kidneys would translate into increased urine levels of $\operatorname{sIg} \mathrm{A}, \operatorname{sIg} \mathrm{A}$ levels were measured in urine and plasma from the same patient cohort as above. Urinary sIgA levels were increased for all diseases, with the highest average level detected for membranous nephropathy (Figure 5A and Table 1). The only exception was minimal

Table 2 Summary of Correlations

\begin{tabular}{lllllc}
\hline Variable & Fibrosis, \% & PIgR, \% & U-sIgA & P-sIgA & P-crea \\
\hline Fibrosis, \% & 1 & $0.76^{* * * *}$ & $0.44^{* *}$ & -0.03 & $0.90^{* * * *}$ \\
PIgR, \% & & 1 & $0.54^{* *}$ & -0.20 & $0.72^{* * * *}$ \\
U-sIgA & & 1 & 0.14 & $0.53^{* *}$ \\
P-sIgA & & & 1 & -0.07 & $0.62^{* * *}$ \\
P-crea & & & & 1 & 0.12 \\
P-urea & & & & $0.70^{* * * *}$ \\
\hline
\end{tabular}

Correlations, as determined by Pearson method, between analyzed patient data, PIgR expression, and extent of kidney fibrosis in samples from the 30 patients in whom tissue was available, as presented in Table 1.

${ }^{*} P<0.05,{ }^{* *} P<0.01,{ }^{* * *} P<0.001$, and ${ }^{* * * *} P<0.0001$.

P-crea, plasma creatinine levels; PIgR, polymeric Ig receptor; P-sIgA, plasma secretory IgA levels; P-urea, plasma urea levels; U-sIgA, urine secretory IgA levels. 


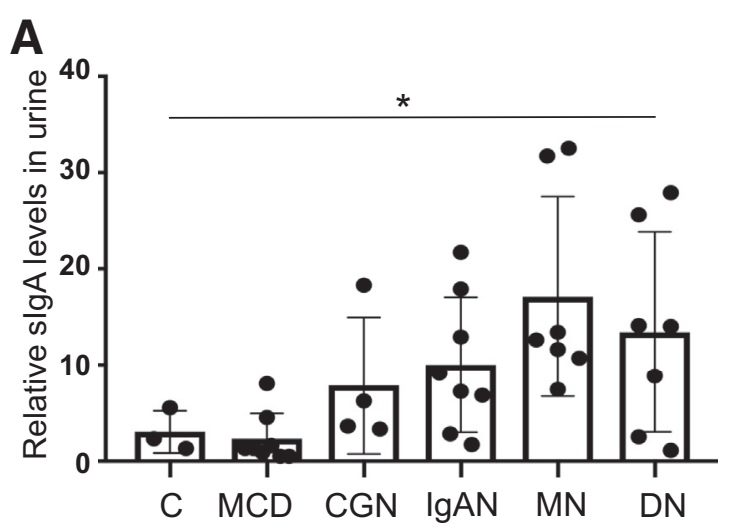

B

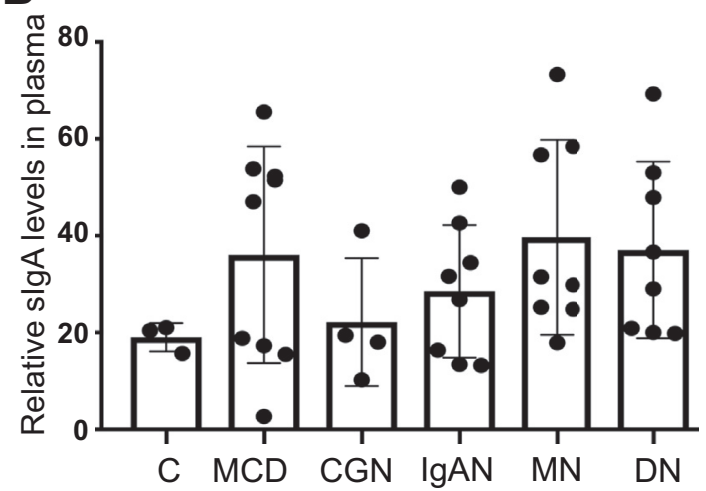

Figure 5 Secretory $\operatorname{IgA}(\operatorname{sg} A)$ levels are elevated in urine and plasma from kidney disease patients. Relative sIgA levels, as determined by sandwich enzyme-linked immunosorbent assay in urine (A) and plasma (B) from healthy controls or from patients with the indicated kidney conditions. Statistical significance was determined by one-way analysis of variance with Dunnett's correction for multiple comparisons. ${ }^{*} P<0.05$. CGN, crescentic glomerulonephritis; C, healthy control; DN, diabetic nephropathy; IgAN, IgA nephropathy; MCD, minimal change disease; MN, membranous nephropathy.

change disease; but more important, this disease is defined by the absence of fibrosis and other histopathologic changes. Not surprisingly, sIgA levels in urine were similar to control values in this category. Interestingly, urinary $\operatorname{sIgA}$ levels correlated significantly to PIgR expression and fibrosis, as well as to plasma creatinine and urea levels (Table 2). This pattern is consistent with the pathology and extent of tubular PIgR expression in the different diseases. A wide range of sIgA levels was observed within patient samples, probably explained by the different degree of structural changes in the kidney caused by age and the severity of the disease. Plasma sIgA levels were elevated compared with healthy controls throughout all of the diseases, including minimal change disease, in which no change in urine sIgA was seen, strongly arguing that increased levels in urine were not simply a reflection of increased levels in plasma (Figure 5B). However, no significant correlations between plasma sIgA levels and PIgR expression or degree of renal injury were found (Table 2). These results indicate that the amount of $\operatorname{sg} \mathrm{A}$ detected in urine is more dependent on PIgR expression level and transport activity in the kidney, rather than on the amount of sIgA present in the blood. Interestingly, a significant correlation between urinary $\operatorname{sIg}$ A levels and increased plasma levels of creatinine and urea, established markers of impaired kidney function, was also observed (Table 2).

\section{Conditioned Medium from Activated Leukocytes Induces PIGR mRNA and Protein in Cultured Human Tubular Cells}

Immune cell infiltrates are commonly seen in injured kidneys. To investigate a potential role of factors secreted from these immune cells in the induction of tubular PIgR expression, primary tubular cells were isolated from human kidney cortex. Both mRNA (Figure 6A) and protein (Figure 6C) levels of PIgR were significantly higher after culture of tubular cells in conditioned medium from human peripheral blood mononuclear cells activated with lipopolysaccharide compared with control medium. A significant increase in PIGR expression at both the mRNA and protein levels was also seen when cells were stimulated with IFN- $\gamma$ (Figure 6, B and C), IL-1 $\beta$ (Figure 6, D and G), and TNF- $\alpha$ (Figure 6, E and G), three cytokines known to be involved in the inflammatory response to injury. Contrarily, treatment with the anti-inflammatory cytokine transforming growth factor- $\beta 1$ resulted in a significant decrease in PIGR mRNA and protein levels (Figure 6, F and $\mathrm{H}$ ). These results suggest that inflammatory cytokines, secreted by leukocytes recruited to the injured kidney, induce the expression of PIgR in proximal tubular epithelial cells.

\section{Cultured Renal Tubular Cells Transport IgA}

To confirm that proximal tubule epithelial cells of the kidney are capable of PIgR-mediated transcytosis of $\mathrm{IgA}$, an in vitro transport assay was performed. A confluent layer of human tubular cells was cultured on permeable membranes in 6-well plates. Isolated human IgA was added to the lower compartment; and after 24 hours, the presence of transported $\operatorname{IgA}$ in the medium, determined as the amount of IgA linked to a secretory component, was analyzed using a sandwich enzymelinked immunosorbent assay. Indeed, secretory $\operatorname{IgA}$ was found in the upper compartment and, furthermore, when cells were pretreated with IFN- $\gamma$ to increase PIgR expression, a significant increase in transported $\operatorname{sIg} \mathrm{A}$ levels was seen (Figure 6I). These results confirm that PIgR, expressed by cultured tubular cells, is functional and capable of performing transcytosis of $\operatorname{IgA}$ by taking up $\operatorname{dIg} \mathrm{A}$ and transporting it across the cell; at the same time, it turns $\operatorname{dIgA}$ into $\operatorname{sIg} \mathrm{A}$ by addition of the secretory component. 
A

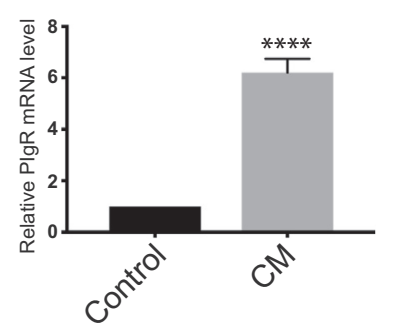

D

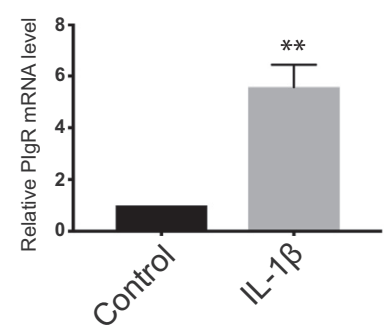

G

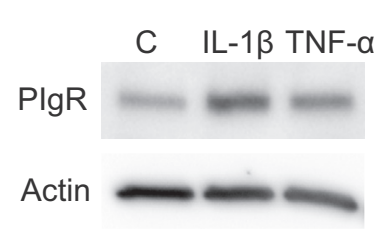

B

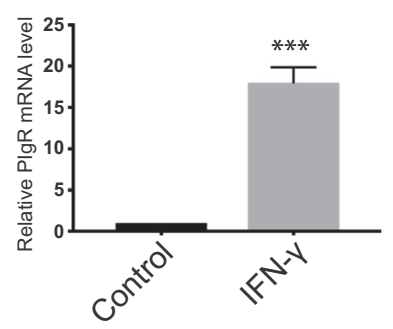

E

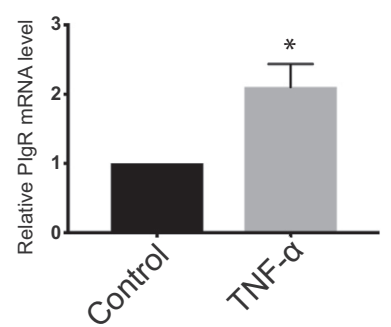

H

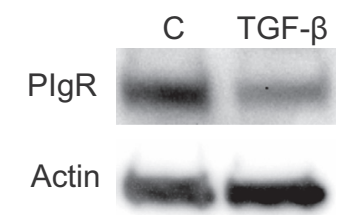

C

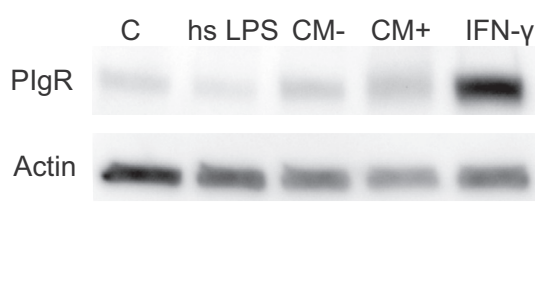

$\mathbf{F}$

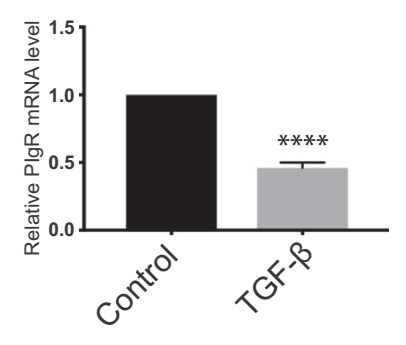

I

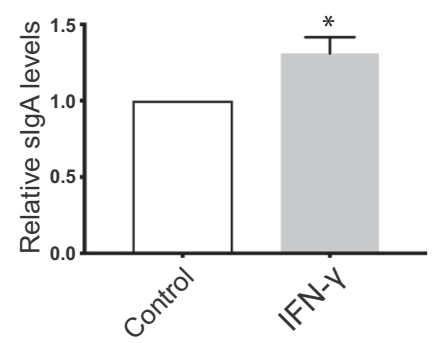

Figure 6 Polymeric Ig receptor (PIgR) expression and IgA transcytosis in cultured tubular cells are induced on exposure to inflammatory cytokines. A-H: Results from real-time quantitative PCR (A, B, and D-F) and Western blot analysis (C, G, and $\mathbf{H})$ showing PIGR mRNA and protein levels in primary human tubular cells after exposure to conditioned medium from activated immune cells or the indicated cytokines for 24 hours. Representative images from Western blot analysis are presented. I: Relative levels of secretory $\operatorname{IgA}(\mathrm{SIgA})$ transported by human tubular cells in vitro, as measured by sandwich enzyme-linked immunosorbent assay after 24 hours of incubation with purified IgA, with or without the addition of interferon- $\gamma$ (IFN- $\gamma$ ). Significance was determined by $t$-test (significant changes of the results compared with control). $n=4(\mathbf{A}, \mathbf{B}$, and $\mathbf{D}-\mathbf{F}) ; n=3(\mathbf{C}, \mathbf{G}$, and $\mathbf{H})$. ${ }^{*} P<0.05,{ }^{* *} P<0.01,{ }^{* * *} P<0.001$, and $* * * * P 0.0001$ versus control. $C$, control medium; CM, immune cell conditioned medium; CM-, nonactivated CM; CM+, activated CM; hs LPS, human serum + lipopolysaccharide; TGF- $\beta$, transforming growth factor- $\beta$; TNF- $\alpha$, tumor necrosis factor- $\alpha$.

\section{PIGR Expression Correlates with the IgA Class Switching Cytokine TNFSF13}

Finally, to get a more comprehensive understanding of PIGR expression, a cross-organ correlation analysis using data from the GTEx portal from eight different tissues with the highest PIGR expression was performed. The mRNA found to have the highest correlation to PIGR mRNA was APRIL (TNFSF13) (Figure 7, A and B), a cytokine involved in B-cell class switching to IgA Igs. Also, TNFRSF11A, involved in $\mathrm{NF}-\kappa \mathrm{B}$ signaling, was among the highest correlating genes. In liver and kidney, the expression of STAT1, which is activated by IFN- $\gamma$, also correlated significantly to $P I G R$ expression. The specific correlation in kidney tissue between the expression levels of PIGR and these genes is demonstrated in Figure 7, B-D. Examples of mRNA with a negative correlation to PIGR levels are shown in Figure 7, E-G. These results point to a connection between an inflammatory phenotype and PIGR expression across human epithelial tissue, including the kidney.

\section{Discussion}

PIgR involvement in $\mathrm{dIgA}$ transport in organs, such as breast, intestine, and respiratory tract, is well established. ${ }^{14,15}$ However, the expression pattern and role of PIgR in the kidney is less studied. Presence of PIgR SC in the human kidney has been demonstrated previously, ${ }^{3,4}$ but the location and regulation have not been clarified. This study shows that in healthy human kidney, PIgR is expressed by parietal epithelial cells of Bowman capsule and, more important, in scattered cells of the proximal tubules. By colocalization to the TSC marker vimentin, the scattered PIgR-positive cells were found to be identical to the previously reported TSCs that have been suggested to be 

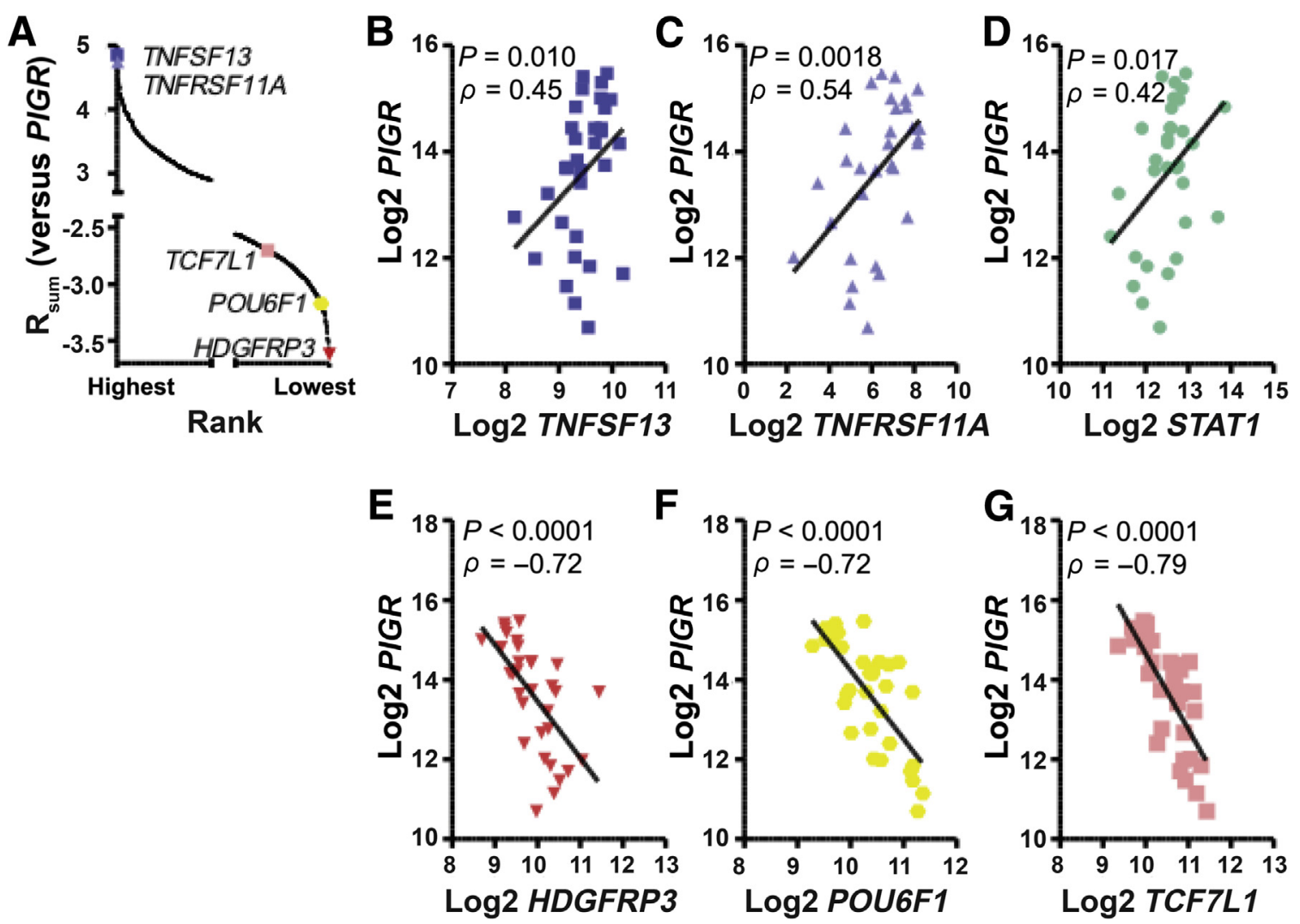

Figure 7 Correlation analysis of polymeric Ig receptor (PIGR) mRNA expression across eight different tissues. Correlation analysis of PIGR expression against transcripts from eight organs showing the highest PIGR expression based on Genotype-Tissue Expression data. A: The sum of correlation coefficients across tissues versus PIGR is sorted in descending order, and the positive and negative extremes are shown. Correlations of interest are highlighted using colored symbols. B-D: The correlations of selected genes (TNFSF13, TNFRSF11A, and STAT1) were next tested in the kidney using the Spearman method. E-G: Examples of negative correlations in the overall analysis in $\mathbf{A}$ that replicate in the kidney data set. $P$ values and Spearman $\rho$ values for the selected correlations are given in the respective panels. Black lines indicate best fit. $n=32$ (B-D).

of importance for the tubular response to injury. ${ }^{5-8} \mathrm{SC}$ has been shown to be expressed in distal tubules in chronically injured kidneys, ${ }^{16}$ and this report shows that as for vimentin, the expression of PIgR becomes extensive in proximal tubules during kidney injury. The colocalization results further demonstrate that in healthy kidney tissue, $80 \%$ of tubular cells positive for PIgR also express vimentin. The degree of colocalization was even higher in diseased kidney tissue. This could indicate that, although both these markers are tightly linked to renal injury, the temporal expression may vary.

In addition, in line with previous reports,${ }^{17-19}$ urinary sIgA levels positively correlated to the degree of kidney injury in patients with kidney disease. Plasma levels of SIgA were also elevated, as has been reported previously for various diseases ${ }^{20-24}$; however, no significant correlation was seen between degree of histologic renal injury and plasma sIgA. In most of the diseases included in this study, both urinary and plasma levels of $\operatorname{sIgA}$ were increased. However, minimal change disease samples deviated in that, although the plasma levels of sIgA were similarly elevated as in the other diseases, the urinary levels remained low. Low immunohistochemical PIgR expression was seen when minimal change disease tissue was evaluated. This likely corresponds to the absence of histologic kidney injury that defines this condition. ${ }^{25,26}$ These results indicate that urinary sIgA levels are more dependent on PIgR expression and activity rather than on plasma IgA levels. Minimal change disease is a disease associated with nephrotic syndrome, characterized by glomerular loss of larger proteins and the low urine levels of $\operatorname{sg} \mathrm{A}$, despite the fact that elevated plasma levels argue against glomerular sIgA leakage.

A significant correlation between urinary $\operatorname{sIg}$ A levels, plasma creatinine, and urea levels as well as degree of renal fibrosis was also found. This indicates a reciprocity between the extent of kidney injury and the sIgA levels in urine, where $\operatorname{sg} \mathrm{A}$ may serve as a marker for kidney injury. Our finding of low basal urinary $\operatorname{sIgA}$ levels in healthy individuals is in line with reports from other groups. ${ }^{17,27,28} \mathrm{~A}$ basal level of sIgA may protect the body from microbial infections, ${ }^{29-31}$ and reduced levels have been suggested to correlate with higher risk of urinary tract infections. ${ }^{32}$ Kidney diseases often result in reduced tubular flow by a reduction in glomerular filtration rate. This could lead to increased risk of ascending urinary tract infection and, thus, it seems likely that PIgR induction in the injured kidney 
may provide increased protection against bacterial infections. In line with this, it has been shown that $\operatorname{sIgA}$ decreases the adhesion of $E$. coli to human urothelial cells. ${ }^{33}$ To investigate PIgR regulation, we drew from the fact that tubular injury almost always is associated with an inflammatory tubulointerstitial cell infiltrate. Exposure of cultured primary tubular cells to conditioned medium from activated human leukocytes resulted in increased mRNA and protein levels of PIgR in the tubular cells. Thus, inflammatory mediators from activated leukocytes may play a role in the regulation of renal PIgR expression. Gastrointestinal expression of PIgR has been shown to be regulated by several proinflammatory cytokines, such as IFN- $\gamma$, TNF- $\alpha$, and IL-1 $\beta .{ }^{15,34}$ Exposing primary tubular cells to these cytokines resulted in a distinct up-regulation of both mRNA and protein levels of PIgR, indicating that renal tubular cells are sensitive to similar cytokines as the more extensively studied gastrointestinal epithelia. TNF- $\alpha$ and IL- $1 \beta$ are strong activators of the NF- $\mathrm{KB}$ signaling cascade, ${ }^{35}$ again underlining that PIgR expression seems to be tightly coupled to response to injury, whereas the antiinflammatory cytokine transforming growth factor- $\beta 1$ instead reduced the expression of PIgR in cultured tubular cells.

Using data from the GTEx portal, ${ }^{12}$ the PIgR gene expression pattern was correlated to the gene expression patterns of other genes across several tissues, including kidney. Interestingly, the top correlation was with TNFSF 13 (alias APRIL). This factor belongs to the TNF superfamily and functions as a key B-cell survival and maturation factor. ${ }^{36}$ TNFSF13 causes class switching to IgA production and also favors the synthesis of $\operatorname{IgA}_{2}$ over $\operatorname{IgA}{ }_{1},{ }^{37}$ with a greater capacity to withstand proteolysis. The GTEx data also show that the kidneys express high levels of TNFSF 13 mRNA compared with other organs. This cytokine could be released during kidney injury and might induce important distant effects on mucosa-associated plasma cells. By occurring in parallel with PIGR induction, this would facilitate the protective effect of SIgA secretion into tubules. A further argument is that the plasma cells infiltrating the tubulointerstitium were found to be negative for JC, indicating that the transported $\operatorname{dIg} \mathrm{A}$ is produced at an extrarenal location.

Thus, we suggest that a basal sIgA excretion into urine is up-regulated during several kidney diseases as part of the TSC phenotype induced by injury of proximal tubules and that PIgR plays a role in the defense against ascending bacterial infections both during normal and disease conditions. Our data show a correlation between urinary sIgA and plasma creatinine or urea levels, further connecting PIgR expression to renal injury.

\section{Acknowledgments}

We thank Kristina Ekström-Holka for skillfully performing immunohistochemical staining.
K.M.K., H.N., D.L., K.L., K.S., and M.E.J. conceptualized the study; K.M.K., H.N., J.N., D.L., K.L., K.S., and M.E.J. designed methods; K.M.K., H.N., J.N., D.L., K.S., and M.E.J. performed experiments; K.M.K., H.N., and M.E.J. wrote the manuscript; M.E.J. acquired funding and supervised the study; all authors read and approved the manuscript, and all authors were involved in revising the manuscript. M.E.J. is the guarantor of this work and, as such, had full access to all the data in the study and takes responsibility for the integrity of the data and the accuracy of the data analysis.

\section{References}

1. Fagarasan S, Honjo T: Intestinal IgA synthesis: regulation of frontline body defences. Nat Rev Immunol 2003, 3:63-72

2. Bienenstock J, Tomasi TB Jr: Secretory gamma-A in normal urine. J Clin Invest 1968, 47:1162-1171

3. Tourville DR, Adler RH, Bienenstock J, Tomasi TB Jr: The human secretory immunoglobulin system: immunohistological localization of gamma A, secretory "piece," and lactoferrin in normal human tissues. J Exp Med 1969, 129:411-429

4. Dobrin RS, Knudson FE, Michael AF: The secretory immune system and renal disease. Clin Exp Immunol 1975, 21:318-328

5. Humphreys BD, Valerius MT, Kobayashi A, Mugford JW, Soeung S, Duffield JS, McMahon AP, Bonventre JV: Intrinsic epithelial cells repair the kidney after injury. Cell Stem Cell 2008, 2:284-291

6. Lindgren D, Bostrom AK, Nilsson K, Hansson J, Sjolund J, Moller C, Jirstrom K, Nilsson E, Landberg G, Axelson H, Johansson ME: Isolation and characterization of progenitor-like cells from human renal proximal tubules. Am J Pathol 2011, 178:828-837

7. Sagrinati C, Netti GS, Mazzinghi B, Lazzeri E, Liotta F, Frosali F, Ronconi E, Meini C, Gacci M, Squecco R, Carini M, Gesualdo L, Francini F, Maggi E, Annunziato F, Lasagni L, Serio M, Romagnani S, Romagnani P: Isolation and characterization of multipotent progenitor cells from the Bowman's capsule of adult human kidneys. J Am Soc Nephrol 2006, 17:2443-2456

8. Smeets B, Boor P, Dijkman H, Sharma SV, Jirak P, Mooren F, Berger K, Bornemann J, Gelman IH, Floege J, van der Vlag J, Wetzels JF, Moeller MJ: Proximal tubular cells contain a phenotypically distinct, scattered cell population involved in tubular regeneration. J Pathol 2013, 229:645-659

9. Vogetseder A, Palan T, Bacic D, Kaissling B, Le Hir M: Proximal tubular epithelial cells are generated by division of differentiated cells in the healthy kidney. Am J Physiol Cell Physiol 2007, 292: C807-C813

10. Krawczyk KM, Hansson J, Nilsson H, Krawczyk KK, Swärd K, Johansson ME: Injury induced expression of caveolar proteins in human kidney tubules: role of megakaryoblastic leukemia 1. BMC Nephrol 2017, 18:320

11. Krawczyk KM, Nilsson H, Allaoui R, Lindgren D, Arvidsson M, Leandersson K, Johansson ME: Papillary renal cell carcinomaderived chemerin, IL-8, and CXCL16 promote monocyte recruitment and differentiation into foam-cell macrophages. Lab Invest 2017, 97:1296-1305

12. Consortium GT: The Genotype-Tissue Expression (GTEx) project Nat Genet 2013, 45:580-585

13. Robinson MD, Oshlack A: A scaling normalization method for differential expression analysis of RNA-seq data. Genome Biol 2010, 11:R25

14. Johansen FE, Kaetzel CS: Regulation of the polymeric immunoglobulin receptor and $\operatorname{IgA}$ transport: new advances in environmental factors that stimulate $\mathrm{pIgR}$ expression and its role in mucosal immunity. Mucosal Immunol 2011, 4:598-602 
15. Kaetzel CS: The polymeric immunoglobulin receptor: bridging innate and adaptive immune responses at mucosal surfaces. Immunol Rev 2005, 206:83-99

16. Abramowsky CR, Swinehart GL: Secretory immune responses in human kidneys. Am J Pathol 1986, 125:571-577

17. Tan Y, Zhang JJ, Liu G, Zhang H, Zhao MH: The level of urinary secretory immunoglobulin A (sIgA) of patients with $\operatorname{IgA}$ nephropathy is elevated and associated with pathological phenotypes. Clin Exp Immunol 2009, 156:111-116

18. Marx M, Weber M, Schafranek D, Wandel E, Meyer zum Buschenfelde KH, Kohler H: Secretory immunoglobulin A in urinary tract infection, chronic glomerulonephritis, and renal transplantation. Clin Immunol Immunopathol 1989, 53:181-191

19. Marx M, Weber M, Kohler H: Urinary secretory immunoglobulin A in acute renal allograft rejection. Nephrol Dial Transplant 1990, 5: $520-524$

20. Frulloni L, Negri M, Brunelli S, Bovo P, Vaona B, Calore B, Liani C, Di Francesco V, Cavallini G: High serum levels of secretory immunoglobullin A in chronic pancreatitis. Dig Liver Dis 2000, 32: 329-334

21. Mole CM, Renoult E, Bene MC, Seilles E, Kessler M, Revillard JP, Faure GC: Serum secretory $\operatorname{IgA}$ and $\operatorname{IgM}$ and free secretory component in IgA nephropathy. Nephron 1995, 71:75-78

22. Seilles E, Rossel M, Vuitton DA, Mercier M, Njoya O, Capron JP, Nalpas B, Gibey R, Revillard JP: Serum secretory IgA and secretory component in patients with non-cirrhotic alcoholic liver diseases. J Hepatol 1995, 22:278-285

23. Waldman RH, Mach JP, Stella MM, Rowe DS: Secretory IgA in human serum. J Immunol 1970, 105:43-47

24. Zhang JJ, Xu LX, Liu G, Zhao MH, Wang HY: The level of serum secretory $\operatorname{IgA}$ of patients with $\operatorname{IgA}$ nephropathy is elevated and associated with pathological phenotypes. Nephrol Dial Transplant 2008, 23:207-212
25. Saha TC, Singh H: Minimal change disease: a review. South Med J 2006, 99:1264-1270

26. Chugh SS, Clement LC, Mace C: New insights into human minimal change disease: lessons from animal models. Am J Kidney Dis 2012, 59:284-292

27. Deo SS, Vaidya AK: Elevated levels of secretory immunoglobulin A (sIgA) in urinary tract infections. Indian J Pediatr 2004, 71:37-40

28. Uehling DT, Johnson DB, Hopkins WJ: The urinary tract response to entry of pathogens. World J Urol 1999, 17:351-358

29. Mazanec MB, Coudret CL, Fletcher DR: Intracellular neutralization of influenza virus by immunoglobulin A anti-hemagglutinin monoclonal antibodies. J Virol 1995, 69:1339-1343

30. Mazanec MB, Kaetzel CS, Lamm ME, Fletcher D, Nedrud JG: Intracellular neutralization of virus by immunoglobulin A antibodies. Proc Natl Acad Sci U S A 1992, 89:6901-6905

31. Yan H, Lamm ME, Bjorling E, Huang YT: Multiple functions of immunoglobulin A in mucosal defense against viruses: an in vitro measles virus model. J Virol 2002, 76:10972-10979

32. Riedasch G, Heck P, Rauterberg E, Ritz E: Does low urinary SIgA predispose to urinary tract infection? Kidney Int 1983, 23: 759-763

33. Svanborg-Eden C, Svennerholm AM: Secretory immunoglobulin A and $\mathrm{G}$ antibodies prevent adhesion of Escherichia coli to human urinary tract epithelial cells. Infect Immun 1978, 22:790-797

34. Johansen FE, Brandtzaeg P: Transcriptional regulation of the mucosal IgA system. Trends Immunol 2004, 25:150-157

35. Lawrence T: The nuclear factor NF-kappaB pathway in inflammation. Cold Spring Harb Perspect Biol 2009, 1:a001651

36. Bossen C, Schneider P: BAFF, APRIL and their receptors: structure, function and signaling. Semin Immunol 2006, 18:263-275

37. Castigli E, Wilson SA, Scott S, Dedeoglu F, Xu S, Lam KP, Bram RJ, Jabara $\mathrm{H}$, Geha RS: TACI and BAFF-R mediate isotype switching in B cells. J Exp Med 2005, 201:35-39 